\title{
Classical and Bayesian Estimation of Two-Parameter Power Function Distribution
}

\author{
Xuechen Liu \\ Faculty of Economics, Taiyuan Normal University 030619, China \\ Email: 1iuxuechenn@126.com \\ Muhammad Arslan \\ School of Statistics, Shanxi University of finance and economics Taiyuan, China \\ Email: arslanma76172@gmail.com \\ Majid Khan \\ Department of Mathematics and Statistics, Riphah International University, Pakistan \\ Email:285004majidkhan@gmail.com \\ Syed Masroor Anwar \\ Department of Statistics, University of Azad Jammu and Kashmir, Pakistan \\ Email: masroorstatistics@gmail.com \\ Zahid Rasheed \\ Department of Mathematics, Women University of Azad Jammu and Kashmir, Pakistan Email: \\ zahidrasheed99@gmail.com
}

\section{*Corresponding author}

Syed Masroor Anwar

Department of Statistics, University of Azad Jammu and Kashmir, Pakistan

Email: masroorstatistics@gmail.com

\begin{abstract}
The power function distribution is a flexible waiting time model that may provide better fit for some failure data. This paper presents the comparison of the maximum likelihood estimates and the Bayes estimates of two-parameter power function distribution. The Bayes estimates are obtained, using conjugate priors, under five loss functions consist of square error, precautionary, weighted, LINEX and DeGroot loss function. The Gibbs sampling algorithm is proposed to generate samples from posterior distributions and in result the Bayes estimates are computed. The comparison of the maximum likelihood estimates and the Bayes estimates are done through the root mean squared errors. One real-life data set is analyzed to illustrate the evaluation of proposed methods of estimation. Finally, results from the simulation are discussed to assess the performance behavior of the maximum likelihood estimates and the Bayes estimates.
\end{abstract}

Keywords: Maximum likelihood estimate; Bayes estimate; Gamma distribution; Squared error loss function; Posterior distribution 


\section{Introduction}

The power function distribution is widely used for semiconductor devices and electrical component reliability. Meniconi and Barry [1] verified that the power function distribution is the best model to test the reliability of an electrical component over exponential, lognormal and Weibull distribution. Zarrin et al. [2] used the power function distribution to estimate the component failure of a semiconductor device. The power function distribution is studied by many authors. For example, Kleiber and Kotz [3] showed that the power function distribution is a particular case of Pareto distribution, Bhatt [4] discussed the characterization of the power function distribution through expectation, Chang [5] considered the power function distribution and discussed its characterizations with the use of independence of record values, Lutful and Ahsanullah [6] used the linear function of the order statistics for estimation of the power function distribution, Malik [7] calculated expressions for the exact moments of order statistics for the power function distribution, Saran and Pandey [8] estimated the power function distribution and its characterizations by kth record value, Saleem et al. [9] derived Bayesian estimators for the finite mixture model of power function distribution with censored sample, Shahzad et al. [10] compared the L-moments method and Trim L-moments methods for the power function distribution and Shakeel et al. [11] used the probability weighted moments method and the generalized probability weighted method to estimate the power function distribution.

This paper presents the comparison of maximum likelihood estimation and Bayesian estimation using a complete sample from the power function distribution. The maximum likelihood and the Bayes estimates for unknown parameters $\theta$ and $\alpha$ are derived and then compared through the root mean squared error. Finally, numeric illustration and comparisons are presented. In the Bayesian estimation problems, it is essential to specify a loss function. In this regard, five loss functions are selected, which consist of squared error loss function (SELF), precautionary loss function (PLF), weighted loss function (WLF), DeGroot loss function (DLF) and LINEX loss function (LLF). The SELF loss function is introduced by Legendre and Gauss in developing the least square theory. This loss function is symmetric and it assign equal weights to positive and negative errors. The PLF is proposed by Norstrom [12]. This loss function is asymmetric loss function and very useful when lower failure rate is under study. The DLF is proposed by DeGroot [13]. This loss function is asymmetric loss function. The LLF is proposed by Varian [14]. This loss function is also asymmetric loss function and preferred to use when there is under estimation is expected. If $\psi$ be the parameter of interest then a list of above-mentioned loss functions with their respective Bayes estimates are given in Table 1. 
Table 1: Bayes estimates under various loss functions.

\begin{tabular}{ll}
\hline Loss Function & Bayes estimate \\
\hline $\operatorname{SELF}=(\psi-\widehat{\psi})^{2}$ & $\widehat{\psi}_{S E L F}=E(\psi \mid \mathbf{x})$ \\
$\operatorname{PLF}=\frac{(\psi-\widehat{\psi})^{2}}{\widehat{\psi}}$ & $\widehat{\psi}_{P L F}=\left(E\left(\psi^{2} \mid \mathbf{x}\right)\right)^{\frac{1}{2}}$ \\
$\mathrm{WLF}=\frac{(\psi-\widehat{\psi})^{2}}{\psi}$ & $\widehat{\psi}_{W L F}=\left(E\left(\psi^{-1} \mid \mathbf{x}\right)\right)^{-1}$ \\
$\mathrm{DLF}=\left(\frac{\psi-\widehat{\psi}}{\widehat{\psi}}\right)^{2}$ & $\widehat{\psi}_{D L F}=\frac{E\left(\psi^{2} \mid \mathbf{x}\right)}{E(\psi \mid \mathbf{x})}$ \\
$\mathrm{LLF}=e^{k(\widehat{\psi}-\psi)}-k(\hat{\psi}-\psi)-1, k \neq 0$ & $\widehat{\psi}_{L L F}=-\frac{1}{k} \ln \left(E\left(e^{-k \psi} \mid \mathbf{x}\right)\right)$ \\
\hline
\end{tabular}

The rest of the paper is outlined as: Section 2 provides the introduction of the power function distribution. Section 3 consists of maximum likelihood estimation for the power function distribution. Section 5 describes the Bayesian estimation. Section 5 presents the Markov chain Monte Carlo (MCMC) technique. Simulation study for the maximum likelihood estimates and the Bayes estimates is conducted in Section 6. A real-life data analysis is performed in Section 7 for illustrative purposes, while a conclusion is given in Section 8.

\section{Power Function Distribution}

The two-parameter power function distribution is defined density function

$$
g(x ; \theta, \alpha)=\frac{\theta x^{\alpha-1}}{\alpha^{\theta}}, 0<x<\alpha, \theta, \alpha>0
$$

where, $\theta$ is a shape parameter, $\alpha$ is a scale parameter. The two-parameter power function distribution is denoted by the notation $\operatorname{PF}(\theta, \alpha)$.

The distribution function of the $P F(\theta, \alpha)$ is given

$$
G(x ; \theta, \alpha)=\left(\frac{x}{\alpha}\right)^{\theta}, 0<x<\alpha, \theta, \alpha>0
$$

The survival function of the $\operatorname{PF}(\theta, \alpha)$ is

$$
S(x ; \theta, \alpha)=1-\left(\frac{x}{\alpha}\right)^{\theta}, \quad 0<x<\alpha, \theta, \alpha>0
$$

Similarly, the hazard function of the $\operatorname{PF}(\theta, \alpha)$ is given by

$$
H(x ; \theta, \alpha)=\frac{\theta x^{\theta-1}}{\alpha^{\theta}-x^{\theta}}, \quad, \quad 0<x<\alpha, \quad \theta, \alpha>0
$$




\section{Power Function Distribution}

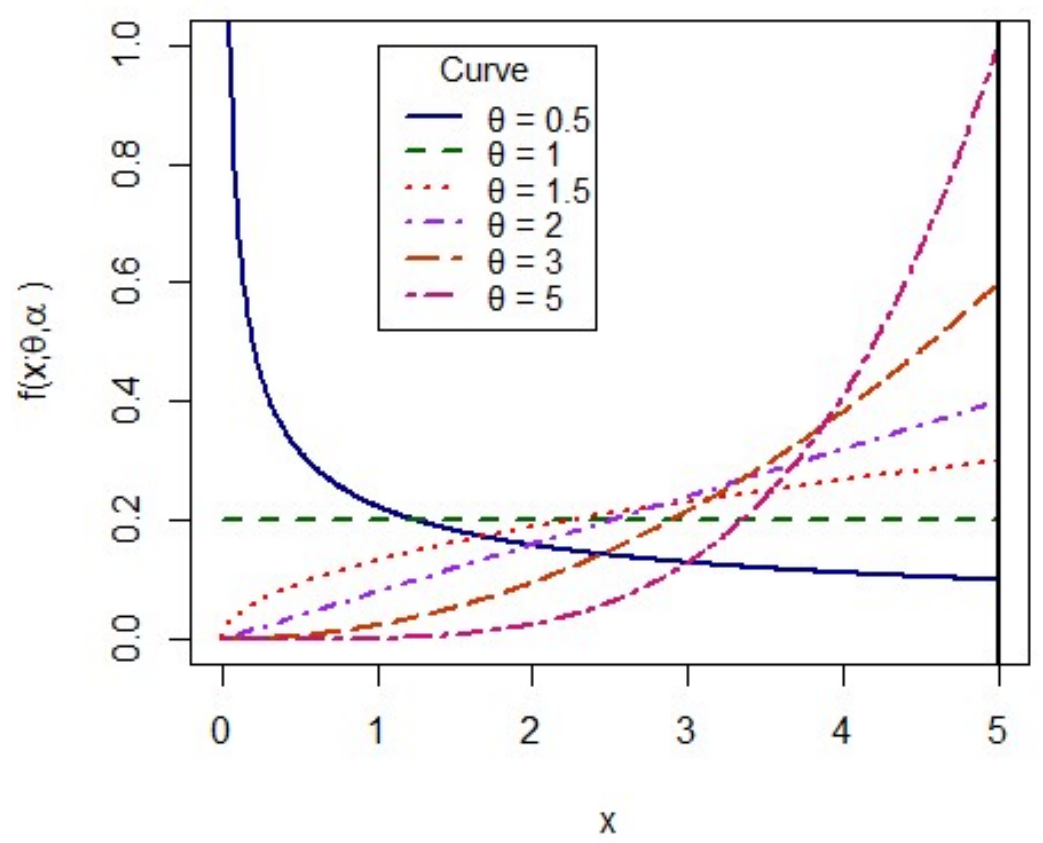

Figure 1. Pdf of power function distribution for different value of $\theta$ at $\alpha=5$

The Figure 1 provides the graphical presentation of the $\operatorname{PF}(\theta, \alpha)$ for different values of $\theta$ at $\alpha=$ 5, which shows that the $\operatorname{PF}(\theta, \alpha)$ is positively skewed and heavy-tailed distribution for $\theta<1$, uniform for $\theta=1$, right triangular for $\theta=2$ and negatively skewed for $\theta>2$.

\section{Maximum Likelihood Estimation}

This section presents maximum likelihood estimates (MLEs) of the $P F(\theta, \alpha)$. Here $\theta$ and $\alpha$ are both assumed unknown. Suppose a random sample of size $n$ is $\mathbf{x}=\left(x_{1}, \ldots, x_{n}\right)^{\prime}$ taken from the PDF in (1) then likelihood function based on $\mathbf{x}$ is given by

$$
L(\theta, \alpha \mid \mathbf{x})=\theta^{n} \alpha^{-n \theta} \prod_{i=1}^{n} x_{i}^{\theta-1}
$$

The log-likelihood function is given by

$$
\ell(\theta, \alpha \mid \mathbf{x})=\log L(\theta, \alpha \mid \mathbf{x})=n \ln (\theta)-n \theta \ln (\alpha)+(\theta-1) \sum_{i=1}^{n} \ln \left(x_{i}\right)
$$

The normal equations are

$$
\begin{aligned}
& \frac{\partial \ell(\theta, \alpha \mid \mathbf{x})}{\partial \theta}=\frac{n}{\theta}-n \ln (\alpha)+\sum_{i=1}^{n} \ln \left(x_{i}\right)=0 \\
& \frac{\partial \ell(\theta, \alpha \mid \mathbf{x})}{\partial \alpha}=-\frac{n \theta}{\alpha}=0
\end{aligned}
$$

The MLE of $\alpha$ is $\hat{\alpha}_{M L E}$ cannot obtained from the equation (8), However the value that maximizes the $\log$-likelihood function for $\alpha$ is the MLE of $\alpha$, given by

$$
\hat{\alpha}_{M L E}=X_{(n)},
$$


where $X_{(n)}$ is the largest order statistic.

The MLE of $\theta$ is $\hat{\theta}_{M L E}$ obtained by solving the equation (5) and (7), given by

$$
\hat{\theta}_{M L E}=\frac{n}{\sum_{i=1}^{n}\left(\ln \left(x_{(n)}\right)-\ln \left(x_{i}\right)\right)}
$$

\section{Bayesian Estimation}

This section introduces the Bayesian analysis of the $P F(\theta, \alpha)$. The Bayesian analysis incorporates the prior knowledge of the parameters through the informative prior densities. If the prior knowledge is not available, non-informative priors are used. Here, the Bayes estimates for unknown parameters $\theta$ and $\alpha$ are derived under their respective independent conjugate priors. The conjugate prior for the unknown parameter $\theta$ is gamma prior, given by

$$
g(\theta)=\frac{b^{a}}{\Gamma(a)} \theta^{a-1} e^{-b}, \quad 0<\theta<\infty,
$$

Similarly, the conjugate prior for unknown parameter $\alpha$ is Pareto prior, given by

$$
g(\alpha)=\frac{c d^{c}}{\alpha^{c+1}}, \quad d<\alpha<\infty
$$

Here $a, b, c, d$ assumed known and non-negative constants, called hyperparameters. The joint prior for $\theta$ and $\alpha$, given by

$$
g(\theta, \alpha) \propto \theta^{a-1} \alpha^{-(c+1)} e^{-b \theta}, \quad 0<\theta<\infty, d<\alpha<\infty
$$

In Bayesian inference, the information about the model parameters is extracted through posterior distribution. According to the Bayes theorem the joint posterior density function of $\theta, \alpha$ given $\mathbf{x}$ defined by

$$
g(\theta, \alpha \mid \mathbf{x})=\frac{L(\theta, \alpha \mid \mathbf{x}) g(\theta, \alpha)}{\int_{0}^{\infty} \int_{T}^{\infty} L(\theta, \alpha \mid \mathbf{x}) g(\theta, \alpha) d \alpha d \theta}
$$

The joint posteriors of $\theta$ and $\alpha$ is obtained as follows,

$$
g(\theta, \alpha \mid \mathbf{x}) \propto \theta^{n+a-1} \alpha^{-(n \theta+c+)} e^{-\left(b+\sum_{i=1}^{n} \ln \left(x_{i}\right)\right) \theta}, \quad 0<\theta<\infty, T<\alpha<\infty,(15
$$

where $T=\operatorname{maximum}\left(b, x_{(n)}\right)$.

Once the posterior distribution is constructed, the next step is to compute the Bayes estimates for any function of $\theta$ and $\alpha$, say $\varphi(\theta, \alpha)$ under various loss functions. The Bayes estimate of $\varphi$ under SELF given by

$$
\hat{\varphi}_{S E L F}=\frac{\int_{0}^{\infty} \int_{T}^{\infty} \varphi \theta^{n+a-1} \alpha^{-(n \theta+c+1)} e^{-\left(b+\sum_{i=1}^{n} \ln \left(x_{i}\right)\right) \theta} d \alpha d \theta}{\int_{0}^{\infty} \int_{T}^{\infty} \theta^{n+a-1} \alpha^{-(n \theta+c+1)} e^{-\left(b+\sum_{i=1}^{n} \ln \left(x_{i}\right)\right) \theta} d \alpha d \theta}
$$

The Bayes estimate of $\varphi$ under PLF is given by 


$$
\hat{\varphi}_{P L F}=\left(\frac{\int_{0}^{\infty} \int_{T}^{\infty} \varphi^{2} \theta^{n+a-1} \alpha^{-(n \theta+c+1)} e^{-\left(b+\sum_{i=1}^{n} \ln \left(x_{i}\right)\right) \theta} d \alpha d \theta}{\int_{0}^{\infty} \int_{T}^{\infty} \theta^{n+a-1} \alpha^{-(n \theta+c+)} e^{-\left(b+\sum_{i=1}^{n} \ln \left(x_{i}\right)\right) \theta} d \alpha d \theta}\right)^{\frac{1}{2}}
$$

The Bayes estimate of $\varphi$ under WLF is given by

$$
\hat{\varphi}_{W L F}=\left(\frac{\int_{0}^{\infty} \int_{T}^{\infty} \varphi^{-1} \theta^{n+a-1} \alpha^{-(n \theta+c+1)} e^{-\left(b+\sum_{i=1}^{n} \ln \left(x_{i}\right)\right) \theta} d \alpha d \theta}{\int_{0}^{\infty} \int_{T}^{\infty} \theta^{n+a-1} \alpha^{-(n \theta+c+1)} e^{-\left(b+\sum_{i=1}^{n} \ln \left(x_{i}\right)\right) \theta} d \alpha d \theta}\right)^{-1}
$$

The Bayes estimate of $\varphi$ under DLF is given by

$$
\hat{\varphi}_{D L F}=\frac{\int_{0}^{\infty} \int_{T}^{\infty} u^{2} \theta^{n+a-1} \alpha^{-(n \theta+c+1)} e^{-\left(b+\sum_{i=1}^{n} \ln \left(x_{i}\right)\right) \theta} d \alpha d \theta}{\int_{0}^{\infty} \int_{T}^{\infty} u \theta^{n+a-1} \alpha^{-(n \theta+c+1)} e^{-\left(b+\sum_{i=1}^{n} \ln \left(x_{i}\right)\right) \theta} d \alpha d \theta}
$$

The Bayes estimate of $\varphi$ under LLF is given by

$$
\hat{\varphi}_{L L F}=-\frac{1}{k} \ln \left(\frac{\int_{0}^{\infty} \int_{T}^{\infty} e^{-k \varphi} \theta^{n+a-1} \alpha^{-(n \theta+c+1)} e^{-\left(b+\sum_{i=1}^{n} \ln \left(x_{i}\right)\right) \theta} d \alpha d \theta}{\int_{0}^{\infty} \int_{T}^{\infty} \theta^{n+a-1} \alpha^{-(n \theta+c+1)} e^{-\left(b+\sum_{i=1}^{n} \ln \left(x_{i}\right)\right) \theta} d \alpha d \theta}\right)
$$

\section{Markov Chain Monte Carlo Technique}

This section consists of a Markov chain Monte Carlo (MCMC) technique, used to generate samples of various sizes from the posterior distributions. Once the MCMC samples are generated, it is feasible to compute the Bayes estimators $\theta$ and $\alpha$ concerning any loss function. The basic MCMC technique is the Gibbs sampler algorithm which frequently used in Bayesian inference. More detail about Gibbs sampler see the Gupta and Singh [15], Papanikolaou et al. [16], Amin [17], Zhang et al. [18],Luengo et al. [19], Adams et al. [20], Gearhart [21] and the references therein. To apply the Gibbs algorithm, the full conditional distributions of the parameters $\theta$ and $\alpha$ are constructed from (14). The conditional density of $\theta$ given $\alpha, \mathbf{x}$ defined by

$$
g(\theta \mid \alpha, \mathbf{x}) \propto \theta^{n+a-1} e^{-\theta\left(b+\sum_{i=1}^{n} \ln \left(x_{i}\right)-\ln (\alpha)\right)}, \quad 0<\theta<\infty, T<\alpha<\infty,(21)
$$

which is a density of gamma distribution with parameters $n+a$ and $b+\sum_{i=1}^{n} \ln \left(x_{i}\right)-\ln (\alpha)$. Similarly, the conditional density of $\alpha$ given $\theta, \mathbf{x}$ defined by

$$
g(\alpha \mid \theta, \mathbf{x}) \propto \alpha^{-(n \theta+c+1)}, \quad 0<\theta<\infty, T<\alpha<\infty,
$$

which is a density of Pareto distribution with parameters $n \theta+c$ and $T=\operatorname{maximum}\left(x_{(n)}, b\right)$. The Gibbs algorithm consists of the following steps

- Step 1: Setting some initial values for $\theta$ and $\alpha$, say $\theta_{0}$ and $\alpha_{0}$, respectively.

- Step 2: Suppose $\theta$ and $\alpha$ take the values $\theta_{j}$ and $\alpha_{j}$ at the $j$ th step, then generating $\theta_{j+1}$ and $\alpha_{j+1}$ from $g\left(\theta \mid \alpha_{j}, \mathbf{x}\right)$ and $g\left(\alpha \mid \theta_{j}, \mathbf{x}\right)$, respectively.

- $\quad$ Step 3: Repeating Step 2, $N$ times.

- Step 4: Calculating Bayes estimate of $\varphi$ by 


$$
\frac{1}{N-M} \sum_{j=M+1}^{N} \hat{\varphi}_{j}
$$

and calculating, root mean squared error (RMSE) by

$$
\sqrt{\frac{1}{N-M} \sum_{j=M+1}^{N}\left(\hat{\varphi}_{j}-\varphi\right)^{2}}
$$

where $M$ is the burn-in period.

\section{Simulation Study}

This section presents a simulation study that is executed to compare the performance behavior of the MLE and the Bayes estimates. The simulation study is carried out for different sample sizes and with different hyperparameter values. In particular, sample of sizes $n=15,25,50$ and 75 are generated by the inverse transformation method from $P F(\theta, \alpha)$. The conjugate priors are used for the estimation of $\operatorname{PF}(\theta, \alpha)$. Two types of Bayes estimates, say Bayes-I estimates and Bayes-II estimates, are computed. The Bayes-I estimates are computed by taking the hyperparameter values $a=0.2, b=1, c=8, d=1$, while the Bayes-II estimates are computed at the hyperparameter values $a=b=c=d=0$, respectively. The set of parametric values that are used for data generation are $(\theta, \alpha) \in\{(0.25,1),(0.5,1.5),(1,2),(1.5,3),(2,5)\}$. The Bayes estimates are computed under SELF, PLF, WLF, DLF, and LLF. The performances of the estimates are compared based on the RMSE. The average estimates along with their RMSE are computed by different methods, given in Table 2 to 5. The RMSE are given in the parentheses below the estimates. All the results in Table 2 to 5 are based on 100000 replications.

\begin{tabular}{|c|c|c|c|c|c|c|c|c|c|c|c|}
\hline \multirow{2}{*}{\multicolumn{2}{|c|}{$\begin{array}{c}(\theta, \alpha) \\
\text { Estimate }\end{array}$}} & \multicolumn{2}{|c|}{$(0.25,1)$} & \multicolumn{2}{|c|}{$(0.5,1.5)$} & \multicolumn{2}{|c|}{$(1,2)$} & \multicolumn{2}{|c|}{$(1.5,3)$} & \multicolumn{2}{|c|}{$(2,5)$} \\
\hline & & $\hat{\theta}$ & $\hat{\alpha}$ & $\hat{\theta}$ & $\hat{\alpha}$ & $\hat{\theta}$ & $\hat{\alpha}$ & $\hat{\theta}$ & $\hat{\alpha}$ & $\hat{\theta}$ & $\hat{\alpha}$ \\
\hline Classical & MLE & $\begin{array}{r}0.2882 \\
(0.0832) \\
\end{array}$ & $\begin{array}{c}0.7893 \\
(0.1698) \\
\end{array}$ & $\begin{array}{r}0.5764 \\
(0.1664) \\
\end{array}$ & $\begin{array}{r}1.3234 \\
(0.1566) \\
\end{array}$ & $\begin{array}{r}1.1528 \\
(0.3328) \\
\end{array}$ & $\begin{array}{r}1.8749 \\
(0.1172) \\
\end{array}$ & $\begin{array}{r}1.7292 \\
(0.4992) \\
\end{array}$ & $\begin{array}{r}2.8722 \\
(0.1221) \\
\end{array}$ & $\begin{array}{r}2.3055 \\
(0.6656) \\
\end{array}$ & $\begin{array}{r}4.8386 \\
(0.1559) \\
\end{array}$ \\
\hline \multirow{5}{*}{ Bayes-I } & SELF & $\begin{array}{c}0.2973 \\
(0.0606)\end{array}$ & $\begin{array}{c}0.4354 \\
(0.0274)\end{array}$ & $\begin{array}{c}0.6862 \\
(0.1407)\end{array}$ & $\begin{array}{c}0.7634 \\
(0.0328)\end{array}$ & $\begin{array}{c}1.2980 \\
(0.2678)\end{array}$ & $\begin{array}{r}1.4407 \\
(0.0416)\end{array}$ & $\begin{array}{c}1.8547 \\
(0.3839)\end{array}$ & $\begin{array}{r}2.4202 \\
(0.0540)\end{array}$ & $\begin{array}{r}2.3648 \\
(0.4906)\end{array}$ & $\begin{array}{r}4.2657 \\
(0.0787) \\
\end{array}$ \\
\hline & PLF & $\begin{array}{r}0.3070 \\
(0.0617) \\
\end{array}$ & $\begin{array}{r}0.4372 \\
(0.0279) \\
\end{array}$ & $\begin{array}{r}0.7088 \\
(0.1434) \\
\end{array}$ & $\begin{array}{r}0.7648 \\
(0.0333) \\
\end{array}$ & $\begin{array}{r}1.3411 \\
(0.2729) \\
\end{array}$ & $\begin{array}{r}1.4419 \\
(0.0420) \\
\end{array}$ & $\begin{array}{r}1.9168 \\
(0.3914) \\
\end{array}$ & $\begin{array}{r}2.4214 \\
(0.0543) \\
\end{array}$ & $\begin{array}{r}2.4443 \\
(0.5001) \\
\end{array}$ & $\begin{array}{r}4.2672 \\
(0.0791) \\
\end{array}$ \\
\hline & WLF & $\begin{array}{r}0.2777 \\
(0.0613) \\
\end{array}$ & $\begin{array}{r}0.4325 \\
(0.0266) \\
\end{array}$ & $\begin{array}{r}0.6405 \\
(0.1423) \\
\end{array}$ & $\begin{array}{c}0.7609 \\
(0.0321)\end{array}$ & $\begin{array}{r}1.2104 \\
(0.2708) \\
\end{array}$ & $\begin{array}{r}1.4385 \\
(0.0410) \\
\end{array}$ & $\begin{array}{r}1.7288 \\
(0.3883) \\
\end{array}$ & $\begin{array}{r}2.4180 \\
(0.0533) \\
\end{array}$ & $\begin{array}{r}2.2035 \\
(0.4961) \\
\end{array}$ & $\begin{array}{r}4.2630 \\
(0.0779) \\
\end{array}$ \\
\hline & DLF & $\begin{array}{c}0.3169 \\
(0.0639)\end{array}$ & $\begin{array}{c}0.4389 \\
(0.0285)\end{array}$ & $\begin{array}{c}0.7320 \\
(0.1484)\end{array}$ & $\begin{array}{c}0.7662 \\
(0.0337)\end{array}$ & $\begin{array}{c}1.3857 \\
(0.2827)\end{array}$ & $\begin{array}{c}1.4431 \\
(0.0424)\end{array}$ & $\begin{array}{r}1.9810 \\
(0.4055)\end{array}$ & $\begin{array}{c}2.4226 \\
(0.0547)\end{array}$ & $\begin{array}{r}2.5264 \\
(0.5182) \\
\end{array}$ & $\begin{array}{r}4.2686 \\
(0.0795) \\
\end{array}$ \\
\hline & LLF & $\begin{array}{r}0.2944 \\
(0.0604)\end{array}$ & $\begin{array}{r}0.4347 \\
(0.0271)\end{array}$ & $\begin{array}{r}0.6710 \\
(0.1402)\end{array}$ & $\begin{array}{c}0.7623 \\
(0.0325)\end{array}$ & $\begin{array}{r}1.2442 \\
(0.2674)\end{array}$ & $\begin{array}{r}1.4390 \\
(0.0412)\end{array}$ & $\begin{array}{r}1.7468 \\
(0.3860) \\
\end{array}$ & $\begin{array}{c}2.4175 \\
(0.0532)\end{array}$ & $\begin{array}{c}2.1923 \\
(0.4978)\end{array}$ & $\begin{array}{c}4.2601 \\
(0.0772)\end{array}$ \\
\hline
\end{tabular}

Table 2: Average estimates and their RMSE (in parentheses) of the $P F(\theta, \alpha)$ for $n=15$ 


\begin{tabular}{|c|c|c|c|c|c|c|c|c|c|c|c|}
\hline \multirow{5}{*}{ Bayes-II } & SELF & $\begin{array}{r}0.3441 \\
(0.0728) \\
\end{array}$ & $\begin{array}{r}0.2935 \\
(0.0543) \\
\end{array}$ & $\begin{array}{r}0.6881 \\
(0.1455) \\
\end{array}$ & $\begin{array}{r}0.8055 \\
(0.0688) \\
\end{array}$ & $\begin{array}{r}1.3762 \\
(0.2910) \\
\end{array}$ & $\begin{array}{r}1.4630 \\
(0.0603) \\
\end{array}$ & $\begin{array}{r}2.0644 \\
(0.4366) \\
\end{array}$ & $\begin{array}{r}2.4346 \\
(0.0662) \\
\end{array}$ & $\begin{array}{r}2.7525 \\
(0.5821) \\
\end{array}$ & $\begin{array}{r}4.2745 \\
(0.0867) \\
\end{array}$ \\
\hline & PLF & $\begin{array}{c}0.3561 \\
(0.0742)\end{array}$ & $\begin{array}{c}0.3101 \\
(0.0619)\end{array}$ & $\begin{array}{c}0.7121 \\
(0.1485)\end{array}$ & $\begin{array}{c}0.8126 \\
(0.0714)\end{array}$ & $\begin{array}{c}1.4243 \\
(0.2969)\end{array}$ & $\begin{array}{r}1.4656 \\
(0.0612)\end{array}$ & $\begin{array}{c}2.1364 \\
(0.4454)\end{array}$ & $\begin{array}{r}2.4365 \\
(0.0668)\end{array}$ & $\begin{array}{r}2.8486 \\
(0.5938)\end{array}$ & $\begin{array}{r}4.2763 \\
(0.0872) \\
\end{array}$ \\
\hline & WLF & $\begin{array}{r}0.3196 \\
(0.0736) \\
\end{array}$ & $\begin{array}{r}0.2784 \\
(0.0496) \\
\end{array}$ & $\begin{array}{c}0.6393 \\
(0.1473) \\
\end{array}$ & $\begin{array}{r}0.7953 \\
(0.0658) \\
\end{array}$ & $\begin{array}{r}1.2786 \\
(0.2945) \\
\end{array}$ & $\begin{array}{r}1.4583 \\
(0.0590) \\
\end{array}$ & $\begin{array}{r}1.9179 \\
(0.4418) \\
\end{array}$ & $\begin{array}{r}2.4312 \\
(0.0652) \\
\end{array}$ & $\begin{array}{r}2.5571 \\
(0.5890) \\
\end{array}$ & $\begin{array}{r}4.2712 \\
(0.0857) \\
\end{array}$ \\
\hline & DLF & $\begin{array}{c}0.3685 \\
(0.0770)\end{array}$ & $\begin{array}{c}0.3276 \\
(0.0721)\end{array}$ & $\begin{array}{c}0.7370 \\
(0.1540)\end{array}$ & $\begin{array}{c}0.8197 \\
(0.0743)\end{array}$ & $\begin{array}{c}1.4740 \\
(0.3080)\end{array}$ & $\begin{array}{c}1.4683 \\
(0.0621)\end{array}$ & $\begin{array}{r}2.2110 \\
(0.4620)\end{array}$ & $\begin{array}{r}2.4384 \\
(0.0674)\end{array}$ & $\begin{array}{r}2.9480 \\
(0.6160)\end{array}$ & $\begin{array}{r}4.2782 \\
(0.0878) \\
\end{array}$ \\
\hline & LLF & $\begin{array}{c}0.3399 \\
(0.0725) \\
\end{array}$ & $\begin{array}{r}0.2895 \\
(0.0528) \\
\end{array}$ & $\begin{array}{c}0.6718 \\
(0.1449) \\
\end{array}$ & $\begin{array}{c}0.8005 \\
(0.0673) \\
\end{array}$ & $\begin{array}{r}1.3131 \\
(0.2909) \\
\end{array}$ & $\begin{array}{r}1.4594 \\
(0.0593) \\
\end{array}$ & $\begin{array}{r}1.9264 \\
(0.4406) \\
\end{array}$ & $\begin{array}{r}2.4304 \\
(0.0650) \\
\end{array}$ & $\begin{array}{r}2.5142 \\
(0.5963) \\
\end{array}$ & $\begin{array}{r}4.2676 \\
(0.0847) \\
\end{array}$ \\
\hline
\end{tabular}

Table 3: Average estimates and their RMSE (in parentheses) of the $P F(\theta, \alpha)$ for $n=25$

\begin{tabular}{|c|c|c|c|c|c|c|c|c|c|c|c|}
\hline \multirow{2}{*}{\multicolumn{2}{|c|}{$\frac{(\theta, \alpha)}{\text { Estimate }}$}} & \multicolumn{2}{|c|}{$(0.25,1)$} & \multicolumn{2}{|c|}{$(0.5,1.5)$} & \multicolumn{2}{|c|}{$(1,2)$} & \multicolumn{2}{|c|}{$(1.5,3)$} & \multicolumn{2}{|c|}{$(2,5)$} \\
\hline & & $\hat{\theta}$ & $\hat{\alpha}$ & $\hat{\theta}$ & $\hat{\alpha}$ & $\hat{\theta}$ & $\hat{\alpha}$ & $\hat{\theta}$ & $\hat{\alpha}$ & $\hat{\theta}$ & $\hat{\alpha}$ \\
\hline Classical & MLE & $\begin{array}{r}0.2717 \\
(0.0578)\end{array}$ & $\begin{array}{c}0.8619 \\
(0.1204) \\
\end{array}$ & $\begin{array}{c}0.5433 \\
(0.1156)\end{array}$ & $\begin{array}{c}1.3887 \\
(0.1037)\end{array}$ & $\begin{array}{c}1.0866 \\
(0.2313)\end{array}$ & $\begin{array}{c}1.9229 \\
(0.0745)\end{array}$ & $\begin{array}{c}1.6300 \\
(0.3469)\end{array}$ & $\begin{array}{c}2.9219 \\
(0.0764)\end{array}$ & $\begin{array}{c}2.1733 \\
(0.4625)\end{array}$ & $\begin{array}{c}4.9018 \\
(0.0968)\end{array}$ \\
\hline \multirow{5}{*}{ Bayes-I } & SELF & $\begin{array}{c}0.2897 \\
(0.0460)\end{array}$ & $\begin{array}{c}0.5989 \\
(0.0305)\end{array}$ & $\begin{array}{c}0.5689 \\
(0.0906)\end{array}$ & $\begin{array}{c}1.1755 \\
(0.0411)\end{array}$ & $\begin{array}{c}1.1059 \\
(0.1768)\end{array}$ & $\begin{array}{c}1.7807 \\
(0.0389)\end{array}$ & $\begin{array}{c}1.6181 \\
(0.2592)\end{array}$ & $\begin{array}{c}2.7821 \\
(0.0448)\end{array}$ & $\begin{array}{c}2.1078 \\
(0.3381)\end{array}$ & $\begin{array}{c}4.7300 \\
(0.0608)\end{array}$ \\
\hline & PLF & $\begin{array}{l}0.2954 \\
(0.0465)\end{array}$ & $\begin{array}{c}0.6004 \\
(0.0310) \\
\end{array}$ & $\begin{array}{c}0.5802 \\
(0.0916) \\
\end{array}$ & $\begin{array}{r}1.1769 \\
(0.0415) \\
\end{array}$ & $\begin{array}{r}1.1280 \\
(0.1789) \\
\end{array}$ & $\begin{array}{r}1.7816 \\
(0.0391) \\
\end{array}$ & $\begin{array}{c}1.6506 \\
(0.2622) \\
\end{array}$ & $\begin{array}{c}2.7828 \\
(0.0450)\end{array}$ & $\begin{array}{r}2.1502 \\
(0.3420) \\
\end{array}$ & $\begin{array}{r}4.7308 \\
(0.0610) \\
\end{array}$ \\
\hline & WLF & $\begin{array}{c}0.2782 \\
(0.0463)\end{array}$ & $\begin{array}{r}0.5962 \\
(0.0298) \\
\end{array}$ & $\begin{array}{r}0.5461 \\
(0.0912) \\
\end{array}$ & $\begin{array}{r}1.1730 \\
(0.0404) \\
\end{array}$ & $\begin{array}{c}1.0612 \\
(0.1780)\end{array}$ & $\begin{array}{c}1.7792 \\
(0.0384)\end{array}$ & $\begin{array}{c}1.5525 \\
(0.2610)\end{array}$ & $\begin{array}{c}2.7808 \\
(0.0444)\end{array}$ & $\begin{array}{c}2.0221 \\
(0.3403)\end{array}$ & $\begin{array}{r}4.7286 \\
(0.0604)\end{array}$ \\
\hline & DLF & $\begin{array}{r}0.3012 \\
(0.0475) \\
\end{array}$ & $\begin{array}{r}0.6020 \\
(0.0315) \\
\end{array}$ & $\begin{array}{c}0.5917 \\
(0.0936) \\
\end{array}$ & $\begin{array}{r}1.1784 \\
(0.0419) \\
\end{array}$ & $\begin{array}{r}1.1505 \\
(0.1827) \\
\end{array}$ & $\begin{array}{r}1.7824 \\
(0.0394) \\
\end{array}$ & $\begin{array}{r}1.6837 \\
(0.2679) \\
\end{array}$ & $\begin{array}{r}2.7835 \\
(0.0452) \\
\end{array}$ & $\begin{array}{r}2.1935 \\
(0.3494) \\
\end{array}$ & $\begin{array}{r}4.7315 \\
(0.0612) \\
\end{array}$ \\
\hline & LLF & $\begin{array}{c}0.2880 \\
(0.0459)\end{array}$ & $\begin{array}{c}0.5980 \\
(0.0302)\end{array}$ & $\begin{array}{c}0.5625 \\
(0.0904)\end{array}$ & $\begin{array}{l}1.1739 \\
(0.0406)\end{array}$ & $\begin{array}{c}1.0819 \\
(0.1766)\end{array}$ & $\begin{array}{c}1.7793 \\
(0.0385)\end{array}$ & $\begin{array}{c}1.5672 \\
(0.2596)\end{array}$ & $\begin{array}{c}2.7803 \\
(0.0442)\end{array}$ & $\begin{array}{c}2.0224 \\
(0.3403)\end{array}$ & $\begin{array}{r}4.7267 \\
(0.0599)\end{array}$ \\
\hline \multirow{5}{*}{ Bayes-II } & SELF & $\begin{array}{c}0.2845 \\
(0.0461) \\
\end{array}$ & $\begin{array}{r}0.6562 \\
(0.0793) \\
\end{array}$ & $\begin{array}{c}0.5689 \\
(0.0921) \\
\end{array}$ & $\begin{array}{r}1.2108 \\
(0.0698) \\
\end{array}$ & $\begin{array}{r}1.1379 \\
(0.1842) \\
\end{array}$ & $\begin{array}{r}1.7955 \\
(0.0507) \\
\end{array}$ & $\begin{array}{r}1.7068 \\
(0.2763) \\
\end{array}$ & $\begin{array}{r}2.7913 \\
(0.0521) \\
\end{array}$ & $\begin{array}{r}2.2758 \\
(0.3684) \\
\end{array}$ & $\begin{array}{r}4.7365 \\
(0.0661) \\
\end{array}$ \\
\hline & PLF & $\begin{array}{c}0.2903 \\
(0.0466) \\
\end{array}$ & $\begin{array}{c}0.6679 \\
(0.0837) \\
\end{array}$ & $\begin{array}{r}0.5806 \\
(0.0932) \\
\end{array}$ & $\begin{array}{r}1.2151 \\
(0.0712) \\
\end{array}$ & $\begin{array}{r}1.1612 \\
(0.1864) \\
\end{array}$ & $\begin{array}{r}1.7969 \\
(0.0511) \\
\end{array}$ & $\begin{array}{r}1.7418 \\
(0.2796) \\
\end{array}$ & $\begin{array}{r}2.7923 \\
(0.0524) \\
\end{array}$ & $\begin{array}{r}2.3224 \\
(0.3728) \\
\end{array}$ & $\begin{array}{r}4.7374 \\
(0.0664) \\
\end{array}$ \\
\hline & WLF & $\begin{array}{r}0.2727 \\
(0.0464) \\
\end{array}$ & $\begin{array}{r}0.6407 \\
(0.0748) \\
\end{array}$ & $\begin{array}{r}0.5454 \\
(0.0927) \\
\end{array}$ & $\begin{array}{r}1.2037 \\
(0.0678) \\
\end{array}$ & $\begin{array}{r}1.0908 \\
(0.1855) \\
\end{array}$ & $\begin{array}{r}1.7928 \\
(0.0499) \\
\end{array}$ & $\begin{array}{r}1.6361 \\
(0.2782) \\
\end{array}$ & $\begin{array}{c}2.7895 \\
(0.0516)\end{array}$ & $\begin{array}{c}2.1815 \\
(0.3710)\end{array}$ & $\begin{array}{c}4.7348 \\
(0.0656)\end{array}$ \\
\hline & DLF & $\begin{array}{c}0.2963 \\
(0.0476) \\
\end{array}$ & $\begin{array}{c}0.6799 \\
(0.0890) \\
\end{array}$ & $\begin{array}{r}0.5925 \\
(0.0953) \\
\end{array}$ & $\begin{array}{r}1.2194 \\
(0.0728) \\
\end{array}$ & $\begin{array}{r}1.1850 \\
(0.1905) \\
\end{array}$ & $\begin{array}{r}1.7983 \\
(0.0515) \\
\end{array}$ & $\begin{array}{r}1.7775 \\
(0.2858) \\
\end{array}$ & $\begin{array}{r}2.7932 \\
(0.0527) \\
\end{array}$ & $\begin{array}{r}2.3700 \\
(0.3810) \\
\end{array}$ & $\begin{array}{r}4.7383 \\
(0.0666) \\
\end{array}$ \\
\hline & LLF & $\begin{array}{c}0.2828 \\
(0.0460)\end{array}$ & $\begin{array}{c}0.6496 \\
(0.0771)\end{array}$ & $\begin{array}{c}0.5623 \\
(0.0919)\end{array}$ & $\begin{array}{c}1.2061 \\
(0.0684)\end{array}$ & $\begin{array}{c}1.1119 \\
(0.1840)\end{array}$ & $\begin{array}{c}1.7930 \\
(0.0500)\end{array}$ & $\begin{array}{c}1.6492 \\
(0.2770)\end{array}$ & $\begin{array}{c}2.7888 \\
(0.0514)\end{array}$ & $\begin{array}{c}2.1748 \\
(0.3717)\end{array}$ & $\begin{array}{c}4.7325 \\
(0.0650)\end{array}$ \\
\hline
\end{tabular}


Table 4: Average estimates and their RMSE (in parentheses) of the $P F(\theta, \alpha)$ for $n=50$

\begin{tabular}{|c|c|c|c|c|c|c|c|c|c|c|c|}
\hline \multirow{2}{*}{\multicolumn{2}{|c|}{$\begin{array}{c}(\theta, \alpha) \\
\text { Estimate }\end{array}$}} & \multicolumn{2}{|c|}{$(0.25,1)$} & \multicolumn{2}{|c|}{$(0.5,1.5)$} & \multicolumn{2}{|c|}{$(1,2)$} & \multicolumn{2}{|c|}{$(1.5,3)$} & \multicolumn{2}{|c|}{$(2,5)$} \\
\hline & & $\hat{\theta}$ & $\hat{\alpha}$ & $\hat{\theta}$ & $\hat{\alpha}$ & $\hat{\theta}$ & $\hat{\alpha}$ & $\hat{\theta}$ & $\hat{\alpha}$ & $\hat{\theta}$ & $\hat{\alpha}$ \\
\hline Classical & MLE & $\begin{array}{r}0.2604 \\
(0.0379) \\
\end{array}$ & $\begin{array}{c}0.9260 \\
(0.0688)\end{array}$ & $\begin{array}{c}0.5208 \\
(0.0758)\end{array}$ & $\begin{array}{r}1.4424 \\
(0.0556)\end{array}$ & $\begin{array}{r}1.0416 \\
(0.1516)\end{array}$ & $\begin{array}{r}1.9608 \\
(0.0385) \\
\end{array}$ & $\begin{array}{c}1.5623 \\
(0.2274)\end{array}$ & $\begin{array}{r}2.9606 \\
(0.0390) \\
\end{array}$ & $\begin{array}{c}2.0831 \\
(0.3032)\end{array}$ & $\begin{array}{r}4.9506 \\
(0.0491)\end{array}$ \\
\hline \multirow{5}{*}{ Bayes-I } & SELF & $\begin{array}{r}0.2428 \\
(0.0274) \\
\end{array}$ & $\begin{array}{c}0.8744 \\
(0.0333)\end{array}$ & $\begin{array}{c}0.4819 \\
(0.0544)\end{array}$ & $\begin{array}{r}1.4116 \\
(0.0336)\end{array}$ & $\begin{array}{c}0.9524 \\
(0.1077)\end{array}$ & $\begin{array}{c}1.9449 \\
(0.0267)\end{array}$ & $\begin{array}{r}1.4137 \\
(0.1599)\end{array}$ & $\begin{array}{r}2.9468 \\
(0.0285)\end{array}$ & $\begin{array}{r}1.8662 \\
(0.2112)\end{array}$ & $\begin{array}{r}4.9350 \\
(0.0371)\end{array}$ \\
\hline & PLF & $\begin{array}{r}0.2453 \\
(0.0275) \\
\end{array}$ & $\begin{array}{r}0.8757 \\
(0.0337) \\
\end{array}$ & $\begin{array}{c}0.4867 \\
(0.0547) \\
\end{array}$ & $\begin{array}{r}1.4124 \\
(0.0338) \\
\end{array}$ & $\begin{array}{r}0.9619 \\
(0.1083) \\
\end{array}$ & $\begin{array}{r}1.9452 \\
(0.0268) \\
\end{array}$ & $\begin{array}{r}1.4279 \\
(0.1609) \\
\end{array}$ & $\begin{array}{r}2.9470 \\
(0.0286) \\
\end{array}$ & $\begin{array}{r}1.8849 \\
(0.2124) \\
\end{array}$ & $\begin{array}{r}4.9352 \\
(0.0372) \\
\end{array}$ \\
\hline & WLF & $\begin{array}{r}0.2380 \\
(0.0274) \\
\end{array}$ & $\begin{array}{c}0.8722 \\
(0.0327)\end{array}$ & $\begin{array}{c}0.4722 \\
(0.0546) \\
\end{array}$ & $\begin{array}{r}1.4102 \\
(0.0332) \\
\end{array}$ & $\begin{array}{c}0.9332 \\
(0.1080)\end{array}$ & $\begin{array}{r}1.9442 \\
(0.0265)\end{array}$ & $\begin{array}{r}1.3852 \\
(0.1605)\end{array}$ & $\begin{array}{c}2.9462 \\
(0.0284)\end{array}$ & $\begin{array}{r}1.8286 \\
(0.2119) \\
\end{array}$ & $\begin{array}{r}4.9345 \\
(0.0370) \\
\end{array}$ \\
\hline & DLF & $\begin{array}{r}0.2477 \\
(0.0278) \\
\end{array}$ & $\begin{array}{c}0.8769 \\
(0.0341) \\
\end{array}$ & $\begin{array}{r}0.4915 \\
(0.0553) \\
\end{array}$ & $\begin{array}{r}1.4132 \\
(0.0341) \\
\end{array}$ & $\begin{array}{r}0.9715 \\
(0.1094) \\
\end{array}$ & $\begin{array}{r}1.9456 \\
(0.0269) \\
\end{array}$ & $\begin{array}{r}1.4422 \\
(0.1626) \\
\end{array}$ & $\begin{array}{r}2.9473 \\
(0.0287) \\
\end{array}$ & $\begin{array}{r}1.9038 \\
(0.2147) \\
\end{array}$ & $\begin{array}{r}4.9355 \\
(0.0373) \\
\end{array}$ \\
\hline & LLF & $\begin{array}{c}0.2423 \\
(0.0273)\end{array}$ & $\begin{array}{c}0.8734 \\
(0.0330)\end{array}$ & $\begin{array}{c}0.4796 \\
(0.0543)\end{array}$ & $\begin{array}{r}1.4106 \\
(0.0333)\end{array}$ & $\begin{array}{c}0.9434 \\
(0.1076)\end{array}$ & $\begin{array}{c}1.9442 \\
(0.0265)\end{array}$ & $\begin{array}{r}1.3939 \\
(0.1600)\end{array}$ & $\begin{array}{r}2.9460 \\
(0.0283)\end{array}$ & $\begin{array}{c}1.8320 \\
(0.2117)\end{array}$ & $\begin{array}{r}4.9337 \\
(0.0368)\end{array}$ \\
\hline \multirow{5}{*}{ Bayes-II } & SELF & $\begin{array}{c}0.2411 \\
(0.0274) \\
\end{array}$ & $\begin{array}{c}0.9077 \\
(0.0601) \\
\end{array}$ & $\begin{array}{c}0.4822 \\
(0.0548) \\
\end{array}$ & $\begin{array}{r}1.4276 \\
(0.0461) \\
\end{array}$ & $\begin{array}{c}0.9643 \\
(0.1095) \\
\end{array}$ & $\begin{array}{r}1.9507 \\
(0.0312) \\
\end{array}$ & $\begin{array}{r}1.4465 \\
(0.1643) \\
\end{array}$ & $\begin{array}{r}2.9503 \\
(0.0313) \\
\end{array}$ & $\begin{array}{r}1.9286 \\
(0.2191) \\
\end{array}$ & $\begin{array}{r}4.9377 \\
(0.0392) \\
\end{array}$ \\
\hline & PLF & $\begin{array}{l}0.2435 \\
(0.0275) \\
\end{array}$ & $\begin{array}{c}0.9119 \\
(0.0614)\end{array}$ & $\begin{array}{c}0.4870 \\
(0.0551)\end{array}$ & $\begin{array}{c}1.4291 \\
(0.0466)\end{array}$ & $\begin{array}{c}0.9741 \\
(0.1102)\end{array}$ & $\begin{array}{r}1.9512 \\
(0.0313)\end{array}$ & $\begin{array}{r}1.4611 \\
(0.1653)\end{array}$ & $\begin{array}{r}2.9506 \\
(0.0314)\end{array}$ & $\begin{array}{c}1.9481 \\
(0.2203)\end{array}$ & $\begin{array}{r}4.9380 \\
(0.0393) \\
\end{array}$ \\
\hline & WLF & $\begin{array}{r}0.2362 \\
(0.0275) \\
\end{array}$ & $\begin{array}{r}0.9009 \\
(0.0582) \\
\end{array}$ & $\begin{array}{r}0.4724 \\
(0.0549) \\
\end{array}$ & $\begin{array}{r}1.4250 \\
(0.0454) \\
\end{array}$ & $\begin{array}{c}0.9447 \\
(0.1099) \\
\end{array}$ & $\begin{array}{r}1.9498 \\
(0.0309) \\
\end{array}$ & $\begin{array}{r}1.4171 \\
(0.1648) \\
\end{array}$ & $\begin{array}{r}2.9497 \\
(0.0311) \\
\end{array}$ & $\begin{array}{r}1.8895 \\
(0.2198) \\
\end{array}$ & $\begin{array}{r}4.9371 \\
(0.0390) \\
\end{array}$ \\
\hline & DLF & $\begin{array}{r}0.2460 \\
(0.0278) \\
\end{array}$ & $\begin{array}{r}0.9161 \\
(0.0629) \\
\end{array}$ & $\begin{array}{r}0.4919 \\
(0.0557) \\
\end{array}$ & $\begin{array}{r}1.4306 \\
(0.0470) \\
\end{array}$ & $\begin{array}{r}0.9839 \\
(0.1114) \\
\end{array}$ & $\begin{array}{r}1.9516 \\
(0.0314) \\
\end{array}$ & $\begin{array}{r}1.4758 \\
(0.1670) \\
\end{array}$ & $\begin{array}{r}2.9510 \\
(0.0315) \\
\end{array}$ & $\begin{array}{r}1.9678 \\
(0.2227) \\
\end{array}$ & $\begin{array}{r}4.9383 \\
(0.0394) \\
\end{array}$ \\
\hline & LLF & $\begin{array}{r}0.2405 \\
(0.0274) \\
\end{array}$ & $\begin{array}{c}0.9042 \\
(0.0590)\end{array}$ & $\begin{array}{c}0.4798 \\
(0.0547)\end{array}$ & $\begin{array}{r}1.4257 \\
(0.0456) \\
\end{array}$ & $\begin{array}{r}0.9550 \\
(0.1094) \\
\end{array}$ & $\begin{array}{r}1.9498 \\
(0.0309) \\
\end{array}$ & $\begin{array}{r}1.4256 \\
(0.1643)\end{array}$ & $\begin{array}{r}2.9494 \\
(0.0310) \\
\end{array}$ & $\begin{array}{r}1.8918 \\
(0.2196) \\
\end{array}$ & $\begin{array}{r}4.9363 \\
(0.0388) \\
\end{array}$ \\
\hline
\end{tabular}

Table 5: Average estimates and their RMSE (in parentheses) of the $P F(\theta, \alpha)$ for $n=75$

\begin{tabular}{|c|c|c|c|c|c|c|c|c|c|c|c|}
\hline \multirow{2}{*}{\multicolumn{2}{|c|}{$\begin{array}{c}(\theta, \alpha) \\
\text { Estimate }\end{array}$}} & \multicolumn{2}{|c|}{$(0.25,1)$} & \multicolumn{2}{|c|}{$(0.5,1.5)$} & \multicolumn{2}{|c|}{$(1,2)$} & \multicolumn{2}{|c|}{$(1.5,3)$} & \multicolumn{2}{|c|}{$(2,5)$} \\
\hline & & \multirow{2}{*}{$\begin{array}{c}\hat{\theta} \\
0.2568 \\
(0.0302)\end{array}$} & \multirow{2}{*}{$\begin{array}{c}\hat{\alpha} \\
0.9496 \\
(0.0480)\end{array}$} & \multirow{2}{*}{$\begin{array}{c}\hat{\theta} \\
0.5136 \\
(0.0604) \\
\end{array}$} & \multirow{2}{*}{$\begin{array}{c}\hat{\alpha} \\
1.4612 \\
(0.0378)\end{array}$} & \multirow{2}{*}{$\begin{array}{c}\hat{\theta} \\
1.0273 \\
(0.1208)\end{array}$} & \multirow{2}{*}{$\begin{array}{c}\hat{\alpha} \\
1.9738 \\
(0.0259)\end{array}$} & \multirow{2}{*}{$\begin{array}{c}\hat{\theta} \\
1.5409 \\
(0.1813) \\
\end{array}$} & \multirow{2}{*}{$\begin{array}{c}\hat{\alpha} \\
2.9737 \\
(0.0261)\end{array}$} & \multirow{2}{*}{$\begin{array}{c}\hat{\theta} \\
2.0546 \\
(0.2417) \\
\end{array}$} & \multirow{2}{*}{$\begin{array}{c}\hat{\alpha} \\
4.9670 \\
(0.0328) \\
\end{array}$} \\
\hline Classical & MLE & & & & & & & & & & \\
\hline \multirow{4}{*}{ Bayes-I } & SELF & $\begin{array}{c}0.2355 \\
(0.0217) \\
\end{array}$ & $\begin{array}{c}0.8646 \\
(0.0256) \\
\end{array}$ & $\begin{array}{r}0.4686 \\
(0.0432) \\
\end{array}$ & $\begin{array}{r}1.3998 \\
(0.0246) \\
\end{array}$ & $\begin{array}{c}0.9303 \\
(0.0858) \\
\end{array}$ & $\begin{array}{r}1.9345 \\
(0.0188) \\
\end{array}$ & $\begin{array}{r}1.3862 \\
(0.1279) \\
\end{array}$ & $\begin{array}{r}2.9352 \\
(0.0198) \\
\end{array}$ & $\begin{array}{r}1.8365 \\
(0.1695) \\
\end{array}$ & $\begin{array}{r}4.9195 \\
(0.0255) \\
\end{array}$ \\
\hline & PLF & $\begin{array}{c}0.2370 \\
(0.0218)\end{array}$ & $\begin{array}{c}0.8653 \\
(0.0259)\end{array}$ & $\begin{array}{r}0.4717 \\
(0.0434) \\
\end{array}$ & $\begin{array}{c}1.4003 \\
(0.0247) \\
\end{array}$ & $\begin{array}{c}0.9366 \\
(0.0862)\end{array}$ & $\begin{array}{r}1.9347 \\
(0.0188) \\
\end{array}$ & $\begin{array}{c}1.3955 \\
(0.1284)\end{array}$ & $\begin{array}{r}2.9353 \\
(0.0198) \\
\end{array}$ & $\begin{array}{c}1.8487 \\
(0.1702)\end{array}$ & $\begin{array}{r}4.9197 \\
(0.0255)\end{array}$ \\
\hline & WLF & $\begin{array}{c}0.2323 \\
(0.0217) \\
\end{array}$ & $\begin{array}{r}0.8632 \\
(0.0253) \\
\end{array}$ & $\begin{array}{r}0.4624 \\
(0.0433) \\
\end{array}$ & $\begin{array}{r}1.3991 \\
(0.0244) \\
\end{array}$ & $\begin{array}{c}0.9179 \\
(0.0860) \\
\end{array}$ & $\begin{array}{r}1.9342 \\
(0.0187) \\
\end{array}$ & $\begin{array}{r}1.3677 \\
(0.1282) \\
\end{array}$ & $\begin{array}{r}2.9349 \\
(0.0197) \\
\end{array}$ & $\begin{array}{r}1.8119 \\
(0.1699) \\
\end{array}$ & $\begin{array}{r}4.9193 \\
(0.0254 \\
\end{array}$ \\
\hline & DLF & $\begin{array}{c}0.2386 \\
(0.0219)\end{array}$ & $\begin{array}{c}0.8660 \\
(0.0261)\end{array}$ & $\begin{array}{c}0.4749 \\
(0.0437)\end{array}$ & $\begin{array}{c}1.4007 \\
(0.0248)\end{array}$ & $\begin{array}{c}0.9428 \\
(0.0868)\end{array}$ & $\begin{array}{r}1.9348 \\
(0.0189)\end{array}$ & $\begin{array}{c}1.4048 \\
(0.1294)\end{array}$ & $\begin{array}{c}2.9354 \\
(0.0199)\end{array}$ & $\begin{array}{c}1.8611 \\
(0.1714)\end{array}$ & $\begin{array}{r}4.9198 \\
(0.0255)\end{array}$ \\
\hline
\end{tabular}




\begin{tabular}{|c|c|c|c|c|c|c|c|c|c|c|c|}
\hline & LLF & $\begin{array}{c}0.2351 \\
(0.0217)\end{array}$ & $\begin{array}{c}0.8639 \\
(0.0255)\end{array}$ & $\begin{array}{c}0.4672 \\
(0.0432)\end{array}$ & $\begin{array}{c}1.3993 \\
(0.0244)\end{array}$ & $\begin{array}{c}0.9246 \\
(0.0858) \\
\end{array}$ & $\begin{array}{c}1.9342 \\
(0.0187)\end{array}$ & $\begin{array}{c}1.3735 \\
(0.1280)\end{array}$ & $\begin{array}{c}2.9348 \\
(0.0197)\end{array}$ & $\begin{array}{c}1.8143 \\
(0.1698)\end{array}$ & $\begin{array}{r}4.9189 \\
(0.0253) \\
\end{array}$ \\
\hline \multirow{5}{*}{ Bayes-II } & SELF & $\begin{array}{r}0.2346 \\
(0.0217) \\
\end{array}$ & $\begin{array}{r}0.8815 \\
(0.0389) \\
\end{array}$ & $\begin{array}{r}0.4691 \\
(0.0434) \\
\end{array}$ & $\begin{array}{r}1.4077 \\
(0.0306) \\
\end{array}$ & $\begin{array}{r}0.9383 \\
(0.0868) \\
\end{array}$ & $\begin{array}{r}1.9373 \\
(0.0209) \\
\end{array}$ & $\begin{array}{r}1.4074 \\
(0.1302) \\
\end{array}$ & $\begin{array}{c}2.9369 \\
(0.0211)\end{array}$ & $\begin{array}{c}1.8766 \\
(0.1736)\end{array}$ & $\begin{array}{r}4.9208 \\
(0.0264)\end{array}$ \\
\hline & PLF & $\begin{array}{c}0.2361 \\
(0.0218)\end{array}$ & $\begin{array}{c}0.8832 \\
(0.0394)\end{array}$ & $\begin{array}{c}0.4723 \\
(0.0436)\end{array}$ & $\begin{array}{c}1.4083 \\
(0.0308)\end{array}$ & $\begin{array}{c}0.9446 \\
(0.0871)\end{array}$ & $\begin{array}{c}1.9375 \\
(0.0210)\end{array}$ & $\begin{array}{c}1.4169 \\
(0.1307)\end{array}$ & $\begin{array}{c}2.9370 \\
(0.0211)\end{array}$ & $\begin{array}{c}1.8891 \\
(0.1743)\end{array}$ & $\begin{array}{r}4.9209 \\
(0.0265)\end{array}$ \\
\hline & WLF & $\begin{array}{r}0.2314 \\
(0.0217) \\
\end{array}$ & $\begin{array}{r}0.8785 \\
(0.0381) \\
\end{array}$ & $\begin{array}{r}0.4628 \\
(0.0435) \\
\end{array}$ & $\begin{array}{r}1.4065 \\
(0.0303) \\
\end{array}$ & $\begin{array}{r}0.9257 \\
(0.0870) \\
\end{array}$ & $\begin{array}{r}1.9368 \\
(0.0208) \\
\end{array}$ & $\begin{array}{r}1.3885 \\
(0.1305) \\
\end{array}$ & $\begin{array}{r}2.9366 \\
(0.0210) \\
\end{array}$ & $\begin{array}{r}1.8513 \\
(0.1740) \\
\end{array}$ & $\begin{array}{r}4.9206 \\
(0.0264) \\
\end{array}$ \\
\hline & DLF & $\begin{array}{r}0.2377 \\
(0.0219) \\
\end{array}$ & $\begin{array}{c}0.8849 \\
(0.0400) \\
\end{array}$ & $\begin{array}{r}0.4755 \\
(0.0439) \\
\end{array}$ & $\begin{array}{r}1.4090 \\
(0.0310) \\
\end{array}$ & $\begin{array}{r}0.9509 \\
(0.0878) \\
\end{array}$ & $\begin{array}{r}1.9377 \\
(0.0210) \\
\end{array}$ & $\begin{array}{r}1.4264 \\
(0.1316) \\
\end{array}$ & $\begin{array}{r}2.9371 \\
(0.0211) \\
\end{array}$ & $\begin{array}{r}1.9018 \\
(0.1755) \\
\end{array}$ & $\begin{array}{r}4.9211 \\
(0.0265) \\
\end{array}$ \\
\hline & LLF & $\begin{array}{c}0.2342 \\
(0.0217)\end{array}$ & $\begin{array}{c}0.8800 \\
(0.0385)\end{array}$ & $\begin{array}{c}0.4677 \\
(0.0434)\end{array}$ & $\begin{array}{c}1.4068 \\
(0.0304)\end{array}$ & $\begin{array}{c}0.9324 \\
(0.0867)\end{array}$ & $\begin{array}{c}1.9369 \\
(0.0208)\end{array}$ & $\begin{array}{c}1.3943 \\
(0.1302)\end{array}$ & $\begin{array}{c}2.9364 \\
(0.0210)\end{array}$ & $\begin{array}{c}1.8533 \\
(0.1739)\end{array}$ & $\begin{array}{r}4.9202 \\
(0.0263)\end{array}$ \\
\hline
\end{tabular}

The performance of the different estimators can be described as

- All the estimates hold the consistency property, i.e., as the sample size increases, the RMSE decreases.

- The Bayes estimates are more efficient than MLE as their RMSE is smaller than the RMSE of MLE.

- The Bayes-I estimates are superior from Bayes-II estimates as the RMSE for Bayes-I is smaller than the RMSE of Bayes-II.

- The Bayes estimates of $\theta$ under LLF are better to those obtained under other loss functions. Similarly, the Bayes estimates of $\alpha$ under weighed loss function are superior to those obtained under other loss functions.

\section{Real Life Data Analysis}

This section provides a real-life data analysis to see how the $P F(\theta, \alpha)$ works in practice. The data set is considered here, used by Ghitany et al. [22], related to a clinical trial performed to study the effectiveness of an antibiotic ointment in relieving pain. This data set represents the 20 failure times (time for a patient to get relief from pain), as follows

$$
\begin{aligned}
& 0.529,0.554,0.566,0.653,0.665,0.683,0.698,0.786,0.788,0.828 \\
& 0.829,0.866,0.879,0.881,0.899,0.917,1.037,1.050,1.110,1.138 \text {. }
\end{aligned}
$$

Before using this data set for the estimation, one natural question arises whether this data set fits the $P F(\theta, \alpha)$ or not. The Kolmogorov-Smirnov (K-S) test is performed for the goodness of fit of the $P F(\theta, \alpha)$. Here, the K-S distance $D_{n}(F)$ is found 0.19912 with a corresponding $p$-value 0.3579 at $\theta=2.02$ and $\alpha=1.18$. As the $p$-value is quite high, which suggests that the $\operatorname{PF}(\theta, \alpha)$ provides good fit for the given failure times data. Thus, there are enough evidence to use this data set for the estimation of $\operatorname{PF}(\theta, \alpha)$. The data set is used to compute the MLEs and the Bayes estimates for the $P F(\theta, \alpha)$.

The MLEs of $\theta$ and $\alpha$ are given as, $\widehat{\theta}_{M L E}=2.8224$ and $\hat{\alpha}_{M L E}=1.1380$, respectively. Similarly, the Bayes estimates of $\theta$ and $\alpha$ are computed under the conjugate priors, where the 
hyperparameters are chosen as $a=0.2, b=1, c=8$ and $d=1$. The Bayes estimates of $\theta$ and $\alpha$ under different loss functions are given as $\left(\hat{\theta}_{S E L F}=2.3928, \hat{\alpha}_{S E L F}=1.1596\right), \quad\left(\hat{\theta}_{P L F}=\right.$ $\left.2.4531, \hat{\alpha}_{P L F}=1.1598\right),\left(\hat{\theta}_{W L F}=2.2708, \hat{\alpha}_{W L F}=1.1592\right),\left(\hat{\theta}_{D L F}=2.5149, \hat{\alpha}_{D L F}=1.1601\right)$ and $\left(\hat{\theta}_{L L F}=2.2577, \hat{\alpha}_{L L F}=1.1594\right)$, respectively. Following the idea of Pradhan and Kundu [23], the comparison among the MLEs, and the Bayes estimates are performed on the basis of the $\mathrm{K}-\mathrm{S}$ test. The K-S test is performed and the K-S distance along with their $p$-value for various estimates are given in Table 6.

Table 6: Kolmogorov-Smirnov distances with their respective $p$-values between the empirical distribution function and the fitted distribution functions

\begin{tabular}{|c|c|c|c|}
\hline & Estimate & K-S Distance & $p$-Value \\
\hline & MLE & 0.2563 & 0.1203 \\
\hline & SELF & 0.2298 & 0.2070 \\
\hline & PLF & 0.2380 & 0.1759 \\
\hline Bayes & WLF & 0.2129 & 0.2838 \\
\hline & DLF & 0.2464 & 0.1483 \\
\hline & LLF & 0.2111 & 0.2919 \\
\hline
\end{tabular}

Therefore, the comparison of the K-S distances showed that the Bayes estimates better fit the $P F(\theta, \alpha)$ as compared to the MLE and among the Bayes estimates under different loss functions, the Bayes estimate under LLF better fit the $\operatorname{PF}(\theta, \alpha)$.

Here, the above real-life data is used to construct the graph of the marginal posterior densities for $\theta$ and $\alpha$.

(a) Posterior distribution of $\theta$

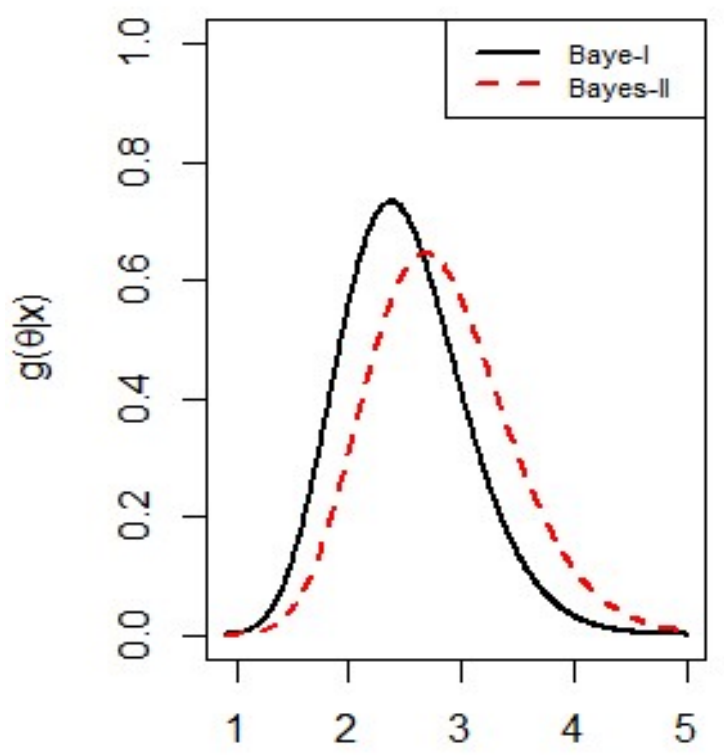

$\theta$ (b) Posterior distribution of $\alpha$

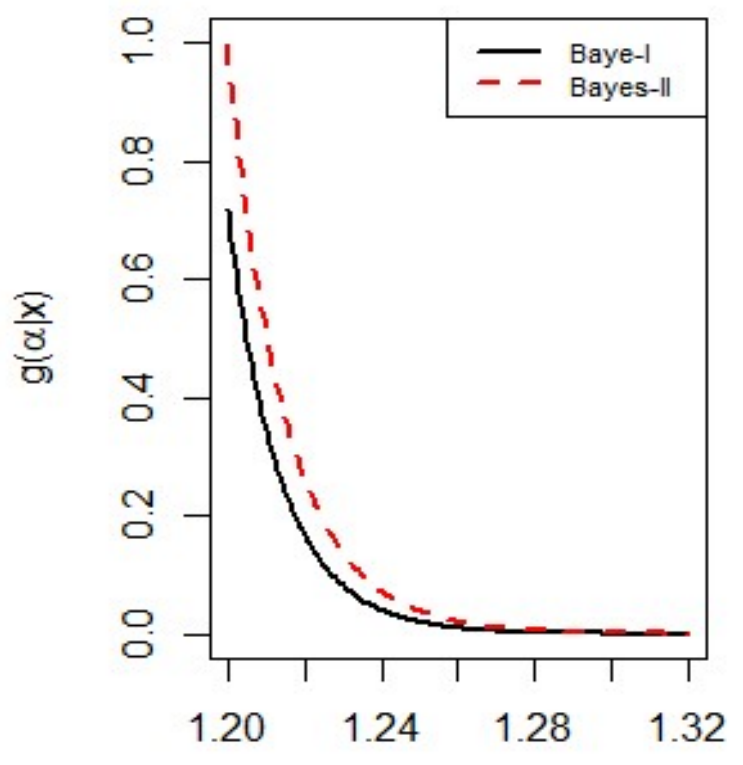

$\alpha$ 
Figure 2. Marginal posterior distributions for $\theta$ and $\alpha$

The Figure 2. represents the graph of the marginal posterior densities for $\theta$ and $\alpha$ at two sets of hyperparameter values $a=0.2, b=1, c=8, d=1$ and $a=b=c=d=0$, repectively. The figure shows that the shapes for marginal posterior densities of $\theta$ are symmetrical, while the shapes for marginal posterior densities for $\alpha$ is heavy-tailed and highly skewed. The figure also shows that the respective marginal posterior distributions for $\theta$ and $\alpha$ under informative and noninformative priors are similar type but fairly sensitive against the hyperparameter values.

\section{Summary and Conclusions}

This paper presents the comparison of maximum likelihood and Bayesian estimation for the unknown parameters of the two-parameter power function distribution. The Bayes estimates are obtained by the Gibbs sampling algorithm, that generate the samples from the posterior distribution of the parameters. The posterior distributions are constructed, using conjugates priors for both parameters $\theta$ and $\alpha$. The conjugate prior for $\theta$ is assumed as gamma prior, and the conjugate prior for $\alpha$ is assumed as Pareto prior. The Bayes estimates are computed under loss functions, consisting of, squared error loss function, precautionary loss function, weighted loss function, DeGroot loss function, and LINEX loss function. The simulation results suggested that the Bayes estimates performed better as compared to maximum likelihood estimate. The simulation results also suggested in the Bayesian estimation, the Bayes estimates under LINEX loss function as compared to the Bayes estimates under other loss functions. The real-life data analysis is suggested the same performance behavior of the estimates as shown by the simulation study.

\section{References}

1 Meniconi, M.,Barry, D. M.: Meniconi M, Barry DM. The Power Function Distribution: A Useful and Simple Distribution to Assess Electrical Component Reliability. Microelectronics Reliability 36, 1207-1212 (1996).

2 Zarrin, S., Saxena, S., Kamal, M.: Reliability computation and Bayesian Analysis of system reliability of Power function distribution. International Journal of Advance in Engineering, Science and Technology 2, 76-86 (2013).

3 Kleiber, C.,Kotz, S.: Statistical Size Distributions in Economics and Actuarial Sciences. John Wiley \& Sons, (2003).

4 Bhatt, M. B.: Characterization of Power-Function Distribution through Expectation. Open Journal of Statistics 3, 441-443.

5 Chang, S.: Characterizations of the Power Function Distribution by the Independence of Record Values. Journal of the Chungcheong Mathematical Society 20, 139-146 (2007).

6 Lutful, K. A.,Ahsanullah, M.: Estimation of the Location and Scale Parameters of a Power Function Distribution by Linear Functions of Order Statistics. Communications in Statistics-Theory and Methods 3, 463-467 (1974).

7 Malik, H. J.: Exact Moments of Order Statistics from a Power Function Distribution. Scandinavian Actuarial Journal, 64-69 (1967).

8 Saran, J.,Pandey, A.: Estimation of Parameters of a Power Function Distribution and its Characterization by k-th Record Values. Statistica 64, 523-536 (2004).

9 Saleem, M., Aslam, M.,Economou, P.: On the Bayesian analysis of the mixture of power function distribution using the complete and the censored sample. Journal of Applied Statistics 37, 25-40 (2010). 
10 Shahzad, M. N., Asghar, Z., Shehzad, F.,Shahzadi, M.: Parameter Estimation of Power Function Distribution with TL-moments. Revista Colombiana de Estadística 38, 321-334 (2015).

11 Shakeel, M., Haq, M. A. U., Hussain, I., Abdulhamid, A. M.,Faisal, M.: Comparison of Two New Robust Parameter Estimation Methods for the Power Function Distribution. PLoS ONE 11 (2016).

12 Norstrom, J. G.: The Use of Precautionary Loss Functions in Risk Analysis. IEEE Trans. Reliab, 45, 400-403 (1996).

13 DeGroot, M. H.: Optimal Statistical Decisions. Vol. 82 John Wiley \& Sons., (2005).

14 Varian, H. R.: A bayesian approach to real estate assessment. Studies in Bayesian econometrics and statistics in honor of Leonard. Journal of Savage, 195-208 (1975).

15 Gupta, P. K.,Singh, A. K.: Classical and Bayesian estimation of Weibull distribution in presence of outliers. Cogent Mathematics 4, 1300975, doi:10.1080/23311835.2017.1300975 (2017).

16 Papanikolaou, Y., Foulds, J. R., Rubin, T. N.,Tsoumakas, G.: Dense Distributions from Sparse Samples: Improved Gibbs Sampling Parameter Estimators for LDA. Journal of Machine Learning Research 18, 1-58 (2017).

17 Amin, A.: Gibbs Sampling for Bayesian Prediction of SARMA Processes. Pakistan Journal of Statistics and Operation Research 15, 397-418 (2019).

18 Zhang, J., Lu, J., Du, H.,Zhang, Z.: Gibbs-Slice Sampling Algorithm for Estimating the Four-Parameter Logistic Model. Frontiers in Psychology 11, doi:10.3389/fpsyg.2020.02121 (2020).

19 Luengo, D., Martino, L., Bugallo, M., Elvira, V.,Särkkä, S.: A survey of Monte Carlo methods for parameter estimation. EURASIP Journal on Advances in Signal Processing 2020, 25, doi:10.1186/s13634-020-00675-6 (2020).

20 Adams, J., Morzfeld, M., Joyce, K., Howard, M.,Luttman, A.: A blocking scheme for dimension-robust Gibbs sampling in large-scale image deblurring. Inverse Problems in Science and Engineering, 1-22, doi:10.1080/17415977.2021.1880398 (2021).

21 Gearhart, C.

22 Ghitany, M. E., Tuan, V. K.,Balakrishnan, N.: Likelihood estimation for a general class of inverse exponentiated distributions based on complete and progressively censored data. Journal of Statistical Computation and Simulation 84, 96-106 (2014).

23 Pradhan, B.,Kundu, D.: Bayes estimation and prediction of the two-parameter gamma distribution. Journal of Statistical Computation and Simulation 81, 1187-1198 (2011). 